\title{
Some dilemmas in energy approach to vibration and stability analysis of pressurized and rotating toroidal shells
}

\author{
Ivo Senjanović( $(1)$, Neven Alujević(1), Ivan Ćatipović(1), Damjan Čakmak(1), Nikola \\ $\operatorname{Vladimir}^{(1)}$, Željan Lozina(2) \\ (1) University of Zagreb, Faculty of Mechanical Engineering and Naval Architecture, Zagreb, CROATIA \\ e-mail:ivo.senjanovic@fsb.hr \\ (2) University of Split, Faculty of Electrical and Mechanical Engineering and Naval Architecture, Split, CROATIA
}

\section{SUMMARY}

In this paper some dilemmas related to the application of the Rayleigh - Ritz method (RRM) in conjunction with the Fourier series for vibration and stability analysis of pressurized and rotating toroidal shells are elucidated. The physical meaning of the strain and kinetic energy terms of different order of displacements in the energy balance equation are explained. Only the second order terms remain in the variation of equation of motion. In the RRM, a symmetric Coriolis mass matrix is obtained as a result of using the energy approach. In FEM, the matrix equation of motion is complex and the Coriolis mass matrix is antisymmetric. It is shown that by transferring the equation of motion from the complex into the real domain, its size is doubled and the total Coriolis matrix becomes symmetric. The influence of using the Green-Lagrange non-linear strains and the engineering strains on vibration and buckling of a toroidal shell is contrasted. It is observed that differences in the dynamic analysis, due to the two different non-linear strain formulations, is quite small. On the contrary, in buckling analysis the engineering strains give considerably higher value of the critical load.

KEY WORDS: toroidal shell; vibration; buckling; energy formulation; geometric stiffness matrix; Coriolis mass matrix.

\section{INTRODUCTION}

Shells are structural elements widely used in engineering structures, such as pressure vessels, cargo tanks on liquefied gas carriers, submarines, offshore structures, engine rotors, automotive tyres etc. Toroidal shells require a special attention due to their wide applicability and geometric complexity. These shells are mainly used as transition elements from cylindrical bodies to spherical heads of thin-walled structures. The toroidal shell theory is rather complicated due to the double curvature of positive and negative sign. It is quite a general 
theory since it comprises theories of complete class of shells of revolution: cylindrical, conical and spherical shells, as well as flat circular membranes and plates, [1-3].

Nowadays, all static and dynamic problems of shell structures are ordinary analysed by the finite element method (FEM), [4,5]. However, this universal method (able to model a wide range of structural geometries) is rather CPU-time consuming. Recently, an analytical solution, the Rayleigh-Ritz method and the finite strip method have been developed for the use in vibration analysis of pressurized and rotating cylindrical and toroidal shells with closed and open cross-sections, [6-10]. An advantage of these methods is a considerable CPU time reduction in comparison to the classical FEM analysis [9]. Vibration analysis of a rotating ring has also been performed by reducing the governing equations of the toroidal shells, [11].

In the energy approach to tackling the problem of vibrations of pressurized and rotating toroidal shells some doubts were observed in [9], which are related to the physical meaning of energy terms of different order of displacements, the symmetry/antisymmetry of the Coriolis mass matrix and the influence of using the so-called engineering strains $[12,13]$ in the analysis of the response of structures considered. A detailed and coherent explanation of the above doubts is given in the present paper.

\section{PHYSICAL MEANING OF STRAIN AND KINETIC ENERGY TERMS OF DIFFERENT ORDER OF DISPLACEMENTS}

For a closed, rotating toroidal shell the displacements components are assumed in the form, [9], Figure 1.

$$
\begin{aligned}
& u(\vartheta, \varphi, t)=U(\vartheta) \cos (n \varphi+\omega t) \\
& v(\vartheta, \varphi, t)=V(\vartheta) \sin (n \varphi+\omega t) \\
& w(\vartheta, \varphi, t)=W(\vartheta) \cos (n \varphi+\omega t)
\end{aligned}
$$

where functions $U(\vartheta), V(\vartheta)$ i $W(\vartheta)$ are the meridional, circumferential and radial displacement components of the cross-section, and $\omega$ is the natural frequency. The unique argument $n \varphi+\omega t$ is introduced in order to capture the travelling modes of a rotating shell.

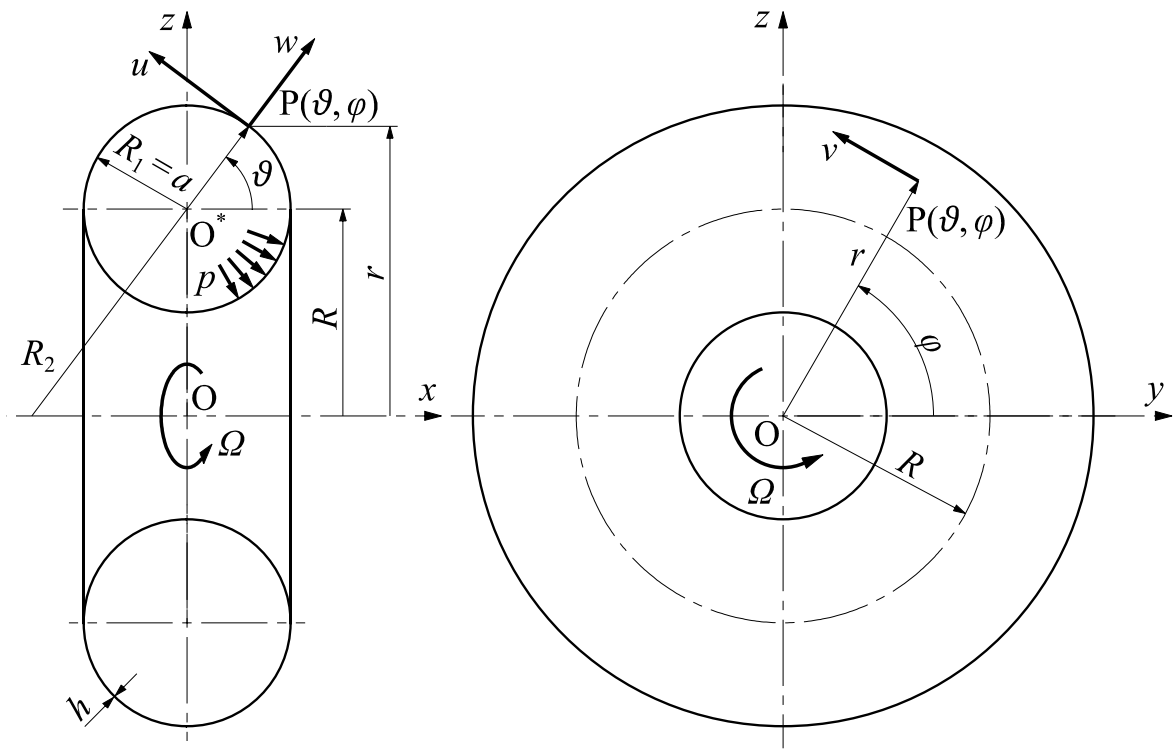

Fig. 1. Geometry and displacements of toroidal shell 
Substituting (1) into the corresponding expressions for strain and kinetic energies and performing integration over the circumferential angle within domain $0-2 \pi$, the temporal variation vanishes and the strain and kinetic energies become time-invariant, [9]. This is due to the fact that the modes rotate while keeping a fixed circumferential profile.

In the case of a rotating toroidal shell the balance of energies reads:

$$
\Pi=E_{s}+E_{G}-E_{k}
$$

where $E_{s}$ is the ordinary linear strain energy, $E_{G}$ is non-linear strain energy due to prestressing, and $E_{k}$ is kinetic energy. For the exact mode shapes, $\Pi$ equals zero. If the energies in (1) are determined for approximated mode shapes, then $\Pi$ is not zero. However, for a successful approximation of the true mode shapes it should be minimum.

According to the classification of the strain and kinetic energies in (1) in terms of different orders of dynamic displacement amplitudes, $\delta^{0}, \delta^{1}, \delta^{2}, \delta^{3}, \delta^{4}$, [9], one can write the differences between the strain and kinetic energies terms in a symbolic form as:

$$
\Pi=\left[E_{G}\left(\delta^{0}\right)-E_{k}\left(\delta^{0}\right)\right]+\left[E_{G}\left(\delta^{1}\right)-E_{k}\left(\delta^{1}\right)\right]+\left[E_{S}\left(\delta^{2}\right)+E_{G}\left(\delta^{2}\right)-E_{k}\left(\delta^{2}\right)\right]+O\left[E_{G}\left(\delta^{3}\right)+E_{G}\left(\delta^{4}\right)\right]
$$

For a rotating closed toroidal shell, pre-stressed with the membrane forces $N_{\vartheta}=0$ and $N_{\vartheta}=\rho h r^{2} \Omega^{2}$, where $\Omega$ is rotation speed, one obtains, [9]:

$$
E_{G}\left(\delta^{0}\right)=\frac{\pi a}{E h} \rho^{2} h^{2} \Omega^{4} \int_{0}^{2 \pi} \tilde{R}^{4} r d \vartheta
$$

where:

$$
\tilde{R}=r+u_{0} \cos \vartheta+w_{0} \sin \vartheta
$$

is the increased value of radius $r$, Figure 1 , due to static displacements, $u_{0}, w_{0}$, caused by the centrifugal load. On the other hand, the kinetic energy reads:

$$
E_{k}\left(\delta^{0}\right)=\pi \rho h a \Omega^{2} \int_{0}^{2 \pi} \tilde{R}^{2} r d \vartheta
$$

The zero order strain energy and kinetic energy represent the energy accumulated in the shell due to rotation:

$$
E_{a k}^{0}=E_{G}\left(\delta^{0}\right)+E_{k}\left(\delta^{0}\right)=\pi \rho h a \Omega^{2}\left[\frac{\rho}{E} \Omega^{2} \int_{0}^{2 \pi} \tilde{R}^{4} r d \vartheta+\int_{0}^{2 \pi} \tilde{R}^{2} r d \vartheta\right]
$$

It is obvious that $E_{G}\left(\delta^{0}\right)-E_{k}\left(\delta^{0}\right)=$ constant for a given $\Omega$.

The first order strain energy due to pre-stressing is obtained in the form:

$$
E_{G}\left(\delta^{1}\right)=2 \pi \rho h a \Omega^{2} \int_{0}^{2 \pi}(U \cos \vartheta+W \sin \vartheta) \tilde{R} r d \vartheta \cos \omega t
$$

Exactly the same expression is derived in [9] for the first order kinetic energy, i.e. $E_{k}\left(\delta^{1}\right)=E_{G}\left(\delta^{1}\right)$.

Matrix equation of motion is obtained by variation of the energy balance equation (3) per displacements. Based on the above consideration the expression in the first brackets in (3) of the $\delta^{0}$ order is constant and its variation is zero. The second term of the $\delta^{1}$ order is a priori zero. Terms of the $\delta^{3}$ and the $\delta^{4}$ order, as a result of non-linear strain energy due to pre- 
stressing, can be ignored as a small quantity in the linearized analysis. Hence, only the terms of the $\delta^{2}$ order remain in (3), and the matrix equation of motion reads:

$$
\frac{\partial \Pi}{\partial\{\delta\}}=\frac{\partial E_{S}\left(\delta^{2}\right)}{\partial\{\delta\}}+\frac{\partial E_{G}\left(\delta^{2}\right)}{\partial\{\delta\}}-\frac{\partial E_{k}\left(\delta^{2}\right)}{\partial\{\delta\}}=\{0\}
$$

\section{MATRIX EQUATION OF MOTION AND SYMMETRIC/ANTISYMMETRIC CORIOLIS MASS MATRIX}

\subsection{APPLICATION OF RAYLEIGH - RITZ METHOD}

In vibration analysis of closed toroidal shell by the Rayleigh - Ritz method displacements are assumed in the form of Fourier series written in matrix notation:

$$
\begin{aligned}
& U(\vartheta)=\left\langle\left\langle f_{m}\right\rangle\left\langle g_{m}\right\rangle\right\rangle\left\{\begin{array}{l}
\left\{A_{m}\right\} \\
\left\{B_{m}\right\}
\end{array}\right\} \\
& V(\vartheta)=\left\langle\left\langle f_{m}\right\rangle\left\langle g_{m}\right\rangle\right\rangle\left\{\begin{array}{l}
\left\{C_{m}\right\} \\
\left\{D_{m}\right\}
\end{array}\right\} \\
& W(\vartheta)=\left\langle\left\langle f_{m}\right\rangle\left\langle g_{m}\right\rangle\right\rangle\left\{\begin{array}{l}
\left\{E_{m}\right\} \\
\left\{F_{m}\right\}
\end{array}\right\}
\end{aligned}
$$

where:

$$
f_{m}=\cos m \vartheta, \quad g_{m}=\sin m \vartheta, \quad m=0,1,2, \ldots, N .
$$

Displacement vector in (9) represents the Fourier coefficients:

$$
\{\delta\}^{T}=\langle\delta\rangle=\left\langle\left\langle A_{m}\right\rangle\left\langle B_{m}\right\rangle\left\langle C_{m}\right\rangle\left\langle D_{m}\right\rangle\left\langle E_{m}\right\rangle\left\langle F_{m}\right\rangle\right\rangle
$$

and the matrix equation of motion is obtained in the form:

$$
\left([K]+p[G]_{p}+\Omega^{2}\left([G]_{\Omega}-[B]\right)-\omega \Omega[C]-\omega^{2}[M]\right)\{\delta\}=\{0\}
$$

where $[K]$ is stiffness matrix, $[G]_{p}$ and $[G]_{\Omega}$ are geometric stiffness matrices due to pressure and rotation, respectively, $[B],[C]$ and $[M]$ are mass matrices due to rotation, Coriolis effect and vibration, respectively. Since the shell theory deals with three displacement components, Eqs. (10), each of the constitutive matrices consists of $3 \times 3=9$ submatrices, i.e.

$$
\begin{aligned}
& {[K]=\left[\begin{array}{lll}
{[K]_{11}} & {[K]_{12}} & {[K]_{13}} \\
{[K]_{21}} & {[K]_{22}} & {[K]_{23}} \\
{[K]_{31}} & {[K]_{32}} & {[K]_{33}}
\end{array}\right]} \\
& {[G]=\left[\begin{array}{lll}
{[G]_{11}} & {[G]_{12}} & {[G]_{13}} \\
{[G]_{21}} & {[G]_{22}} & {[G]_{23}} \\
{[G]_{31}} & {[G]_{32}} & {[G]_{33}}
\end{array}\right],[G]=p[G]_{p}+\Omega^{2}[G]_{\Omega}}
\end{aligned}
$$




$$
\begin{gathered}
{[B]=\left[\begin{array}{ccc}
{[B]_{11}} & {[0]} & {[B]_{13}} \\
{[O]} & {[B]_{22}} & {[O]} \\
{[B]_{31}} & {[0]} & {[B]_{33}}
\end{array}\right]} \\
{[C]=\left[\begin{array}{ccc}
{[0]} & {[C]_{12}} & {[0]} \\
{[C]_{21}} & {[0]} & {[C]_{23}} \\
{[O]} & {[C]_{32}} & {[0]}
\end{array}\right]} \\
{[M]=\left[\begin{array}{ccc}
{[M]_{11}} & {[0]} & {[0]} \\
{[O]} & {[M]_{22}} & {[0]} \\
{[O]} & {[O]} & {[M]_{33}}
\end{array}\right]}
\end{gathered}
$$

All the above matrices are symmetric; including the Coriolis mass matrix $[C]$, with the zero main diagonal submatrices, i.e. elements.

\subsection{APPLICATION OF THE FINITE ELEMENT METHOD}

In the finite element method (FEM) displacement field of a finite element is assumed in the form:

$$
\{\delta(x, y, z, t)\}^{e}=[N(x, y, z)]\{\Delta(t)\}^{e}
$$

where $[N(x, y, z)]$ is the matrix of shape functions and $\{\Delta(t)\}^{e}$ is the vector of nodal displacements. By assembling finite elements, the matrix differential equation of motion of complete structure is obtained in the form, [14]:

$$
[M]\{\ddot{q}\}+\Omega[C]\{\dot{q}\}+\left([K]+p[G]_{p}+\Omega^{2}\left([G]_{\Omega}-[B]\right)\right)\{q\}=\{0\}
$$

where $\{q\}$ is the vector of nodal displacements. In Eq. (19) all matrices are symmetric except the Coriolis matrix which is an antisymmetric one. Since natural vibrations are harmonic one can write:

$$
\{q(t)\}=\{\delta\} e^{i \omega t}
$$

and the time variable is eliminated from (19). This yields:

$$
\left([K]+p[G]_{p}+\Omega^{2}\left([G]_{\Omega}-[B]\right)+i \omega \Omega[C]-\omega^{2}[M]\right)\{\delta\}=\{0\}
$$

where $i=\sqrt{-1}$.

By comparing Eq. (13) to Eq. (22) it can be seen that the structure of both equations is similar. The difference, however, is the imaginary unit in (22) and the antisymmetric matrix $[C]$. It is well known that the Coriolis matrix causes bifurcation of natural frequencies. Numerical examples show that RRM and FEM give very similar results, Figs. 2 and 3, [9]. The question is how can this be possible given the fact that Coriolis matrix is on one occasion symmetric and on the other antisymmetric? The explanation is given below.

In FEM the eigenvalue problem (22) is formulated in the complex domain, and consequently modal displacements consist of real and imaginary parts:

$$
\{\delta\}=\{\delta\}_{R e}+i\{\delta\}_{\text {Im }}
$$


Substituting (23) into (22) one obtains for the real part:

$$
\left[\begin{array}{cc}
{[K(\omega)]} & -\omega \Omega[C] \\
\omega \Omega[C] & {[K(\omega)]}
\end{array}\right]\left\{\begin{array}{l}
\{\delta\}_{R e} \\
\{\delta\}_{\text {Im }}
\end{array}\right\}=\{0\}
$$

where:

$$
[K(\omega)]=[K]+p[G]_{p}+\Omega^{2}\left([G]_{\Omega}-[B]\right)-\omega^{2}[M]
$$

is the dynamic stiffness matrix. Observing the total matrix in (25), the first impression is that it is antisymmetric. However, since $[C]$ is an antisymmetric matrix, the following identity is valid:

$$
[C]=-[C]^{T}
$$

Substituting this relation into one of two $[C]$ terms in (24) it is obvious that the resulting total Coriolis matrix formulated in the real domain is symmetric.

If Coriolis matrix $[C]$ is ignored in (24) a set of double frequencies is obtained. By taking Coriolis matrix into account, these frequencies are bifurcated into two values related to the forward and backward travelling mode profiles, Figures 2 and 3.

In RRM a symmetric Coriolis matrix is obtained directly in the real domain as a result of the energy approach formulation. The above facts are illustrated next by two simple examples.

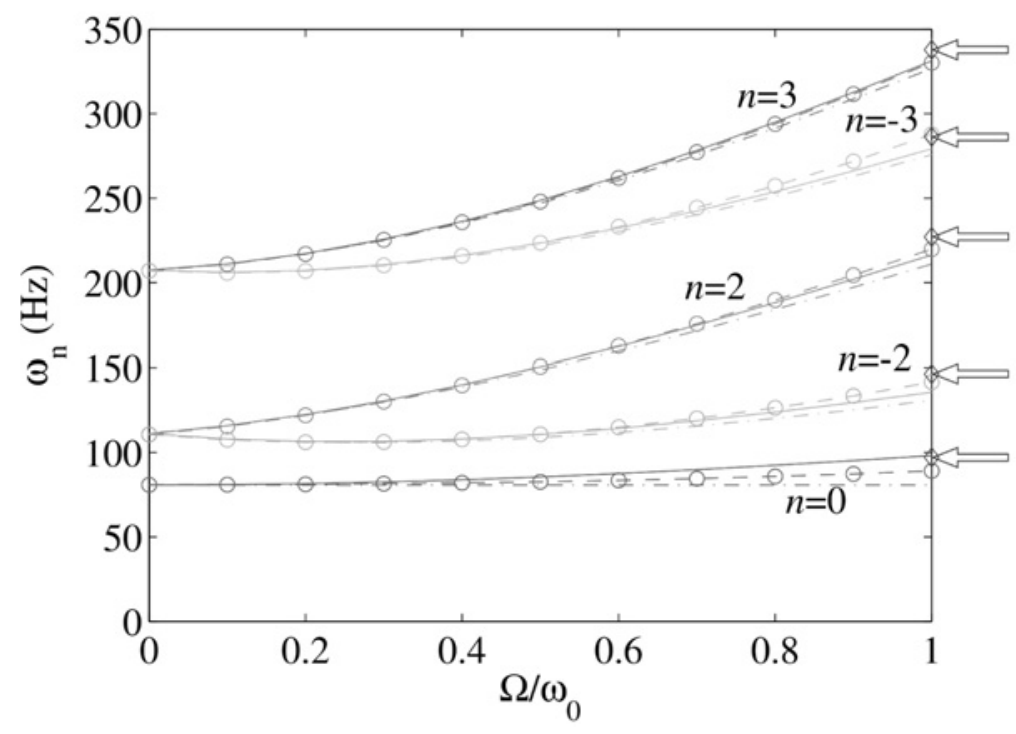

Fig. 2 Natural frequencies of rotating toroidal shell, $R=1 \mathrm{~m}, a=0.4 \mathrm{~m}, \mathrm{~h}=0.01 \mathrm{~m}$, asymmetric modes: --- RRM, tension forces - shell theory, - - RRM, tension forces - membrane theory, - o - FEM, $\Leftarrow R R M$, eng. strains, Ref. [9] 


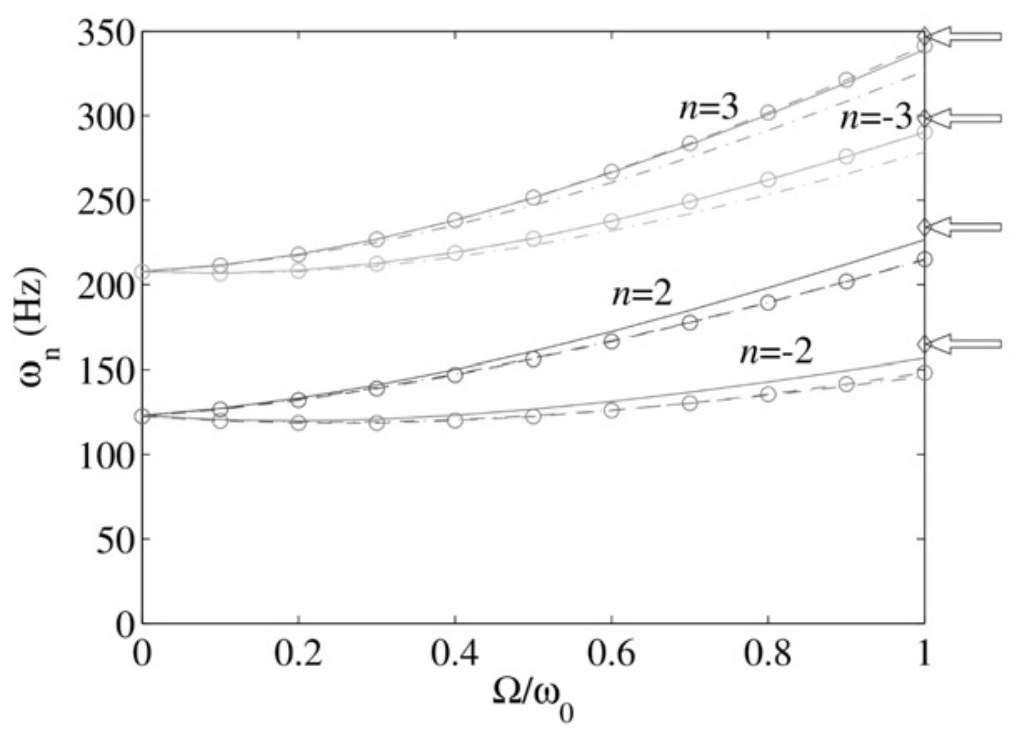

Fig. 3 Natural frequencies of rotating toroidal shell, $R=1 \mathrm{~m}, a=0.4 \mathrm{~m}, h=0.01 \mathrm{~m}$, symmetric modes: --- RRM, tension forces - shell theory, - - RRM, tension forces - membrane theory, - o - FEM, $\Leftarrow R R M$, eng. strains, Ref. [9]

\subsection{RING IN-PLANE VIBRATIONS}

A ring performing in-plane vibrations can be considered as a short top segment of toroidal shell with displacements $V$ and $W$, Figure 4 .

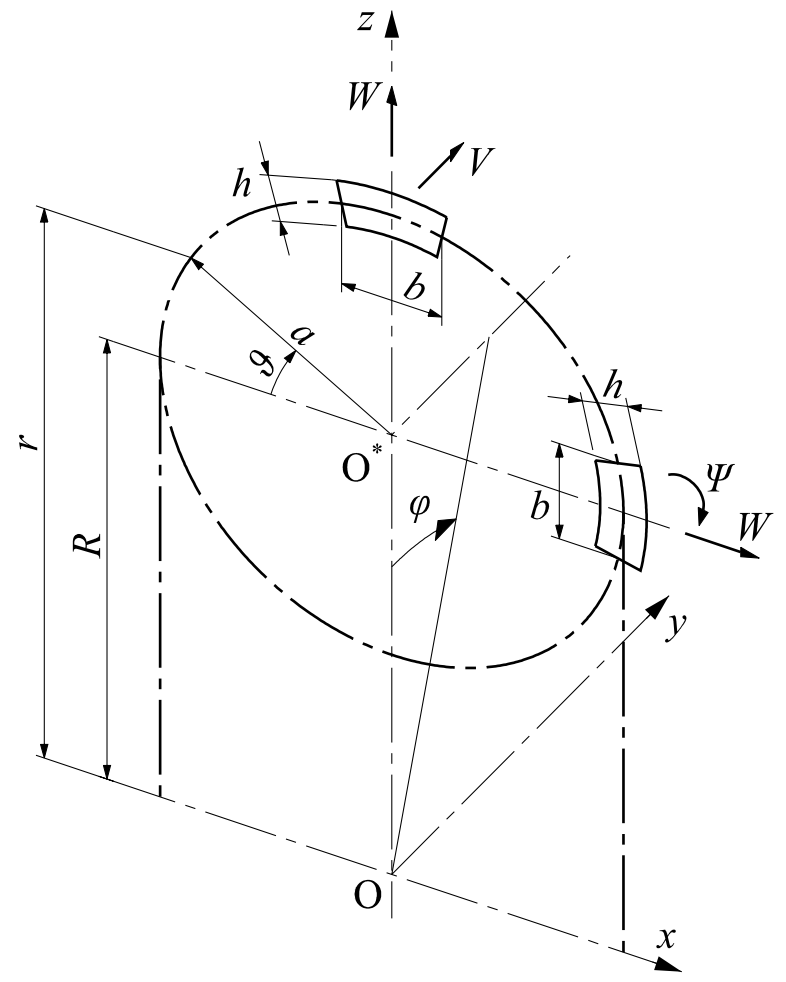

Fig. 4 Rings as toroidal shell segments 
The governing matrix equation of motion is derived in [11] by a deduction from a general toroidal shell theory:

$$
\left[\begin{array}{cc}
a_{11}-\omega^{2} & a_{12}-2 \omega \Omega \\
a_{21}-2 \omega \Omega & a_{22}-\omega^{2}
\end{array}\right]\left\{\begin{array}{l}
V \\
W
\end{array}\right\}=\{0\}
$$

where:

$$
\begin{aligned}
& a_{11}=\frac{p_{5}}{\alpha}+\frac{c_{4}}{\alpha}-\Omega^{2} \\
& a_{22}=\frac{q_{3}}{\alpha}+\frac{c_{7}}{\alpha}-\Omega^{2} \\
& a_{12}=a_{21}=\frac{q_{16}}{\alpha}+\frac{c_{11}}{\alpha}
\end{aligned}
$$

The above coefficients are the following:

$$
\begin{aligned}
& \frac{p_{5}}{\alpha}=\frac{K}{\rho h r^{2}} n^{2}\left(1+\frac{D}{K r^{2}}\right) \\
& \frac{q_{3}}{\alpha}=\frac{K}{\rho h r^{2}}\left(1+n^{4} \frac{D}{K r^{2}}\right) \\
& \frac{q_{16}}{\alpha}=\frac{K}{\rho h r^{2}} n\left(1+n^{2} \frac{D}{K r^{2}}\right) \\
& \frac{c_{4}}{\alpha}=\frac{c_{7}}{\alpha}=\left(n^{2}+1\right) \Omega^{2} \\
& \frac{c_{11}}{\alpha}=2 n \Omega^{2}
\end{aligned}
$$

Eq. (27) can be presented in the extended form (12), where, without taking the pressure into account:

$$
\begin{aligned}
& {[K]=\frac{1}{\alpha}\left[\begin{array}{cc}
p_{5} & q_{16} \\
q_{16} & q_{3}
\end{array}\right], \quad[G]_{\Omega}=\left[\begin{array}{cc}
n^{2}+1 & 2 n \\
2 n & n^{2}+1
\end{array}\right]} \\
& {[B]=\left[\begin{array}{ll}
1 & 0 \\
0 & 1
\end{array}\right], \quad[C]=2\left[\begin{array}{ll}
0 & 1 \\
1 & 0
\end{array}\right], \quad[M]=\left[\begin{array}{ll}
1 & 0 \\
0 & 1
\end{array}\right]}
\end{aligned}
$$

The symmetry of the Coriolis matrix [C] is obvious. In reference [11] Eq. (27) is expanded into a polynomial of the fourth order and solved analytically in a rigorous way, approximately, as well as numerically.

\subsection{WHIRLIGIG OSCILLATIONS}

A simple model leading to the form of the FEM eigenvalue formulation, Eq. (20), is the whirligig shown in Figure 5. It consists of a simply supported disk and elastically supported shaft end. Whirligig matrix differential equation of motion is obtained by considering equilibrium of inertia moments, Coriolis moments and restoring moments. Hence, one can write:

$$
J_{a}\left[\begin{array}{ll}
1 & 0 \\
0 & 1
\end{array}\right]\left\{\left\{\begin{array}{l}
\{\ddot{\varphi}\} \\
\{\ddot{\psi}\}
\end{array}\right\}+\Omega J_{p}\left[\begin{array}{cc}
0 & -1 \\
1 & 0
\end{array}\right]\left\{\left\{\begin{array}{l}
\dot{\varphi}\} \\
\{\dot{\psi}\}
\end{array}\right\}+\left[\begin{array}{cc}
K_{11} & 0 \\
0 & K_{22}
\end{array}\right]\left\{\begin{array}{l}
\{\varphi\} \\
\{\psi\}
\end{array}\right\}=\{0\}\right.\right.
$$


where $J_{a}=\rho I_{x x}=\rho I_{y y}$ is the disk mass moment of inertia, $J_{p}=\rho I_{p}$ is the disk mass polar moment of inertia, $K_{11}=I^{2} k_{22}$ and $K_{22}=I^{2} k_{11}$ are the angular stiffnesses of elastic supports, and $\varphi$ and $\psi$ are slope angles of the rigid shaft. The antisymmetry of the Coriolis matrix in (31) can be noticed. Taking into account:

$$
\left\{\begin{array}{c}
\varphi \\
\psi
\end{array}\right\}=\left\{\begin{array}{c}
\Phi_{R e}+i \Phi_{I m} \\
\Psi_{R e}+i \Psi_{I m}
\end{array}\right\} e^{i \omega t}
$$

the eigenvalue formulation in the real domain according to (24) reads:

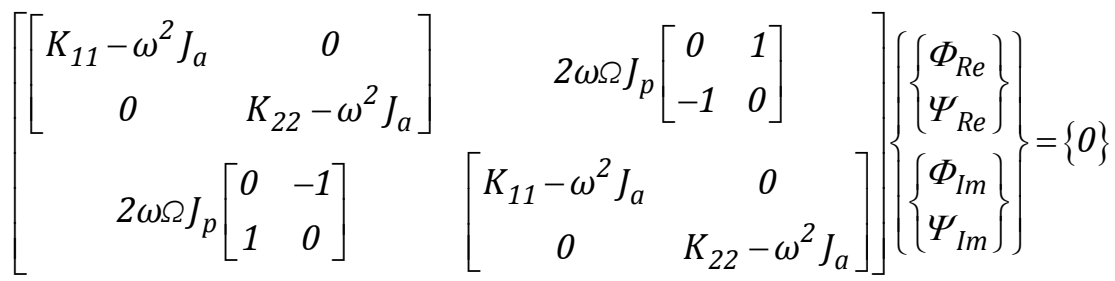

The symmetry of the system (33) is evident. The determinant of Eq. (33) can be expanded into a polynomial of the fourth order.

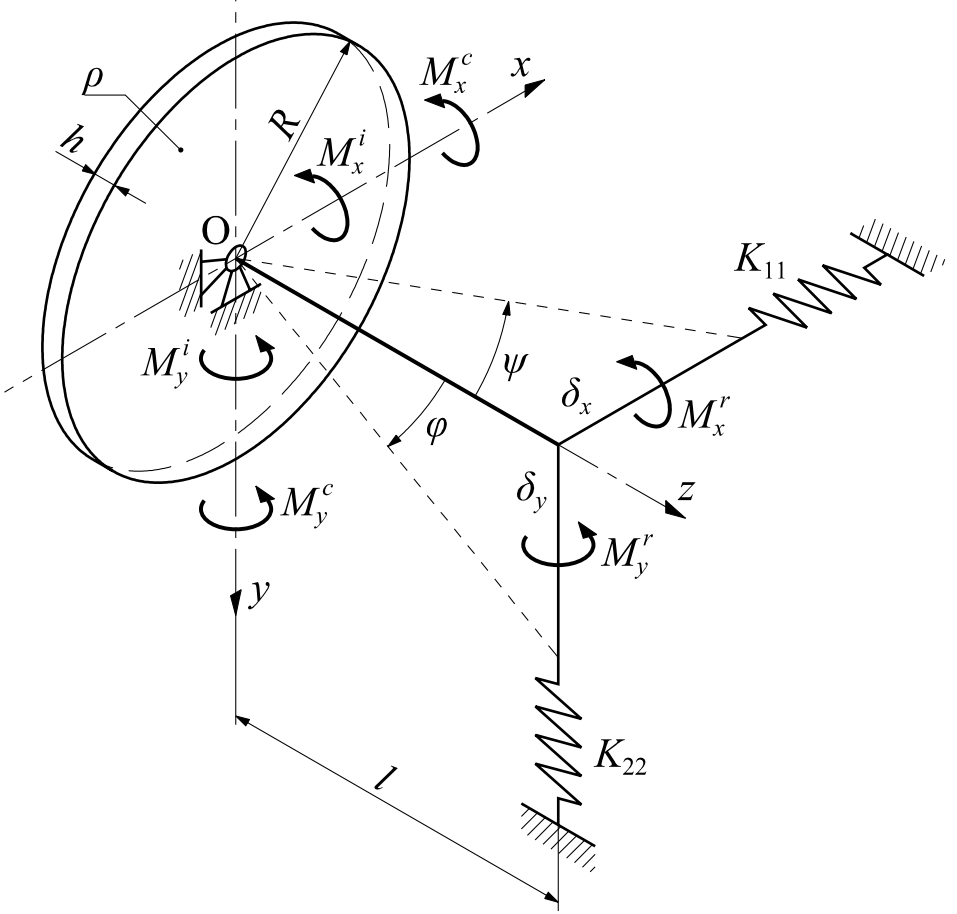

Fig. 5 Whirligig system, displacements and moments

\subsection{FORMAL SIMILARITY AND DIFFERENCE BETWEEN MATHEMATICAL MODELS FOR ROTATING DYNAMIC SYSTEM AND STATIONARY DAMPED DYNAMIC SYSTEM}

General formulation of matrix differential equation of motion is common for both rotating and stationary damped dynamic system, i.e.

$$
[M]\{\ddot{q}\}+[C]\{\dot{q}\}+[K]\{q\}=\{0\}
$$


Matrix $[C]$ in the former case is the Coriolis mass matrix, $[C]_{c}$, and in the latter case the damping matrix $[C]_{d}$. As stated in the previous section, $[C]_{c}$ is antisymmetric matrix with obvious zero elements on the main diagonal. Damping matrix $[C]_{d}$ is usually scarcely populated with at least one main diagonal element different from zero.

The solution of Eq. (34) can be assumed in the exponential form:

$$
\{q(t)\}=\{\delta\} e^{\lambda t}
$$

In this way the time variable is eliminated from (34) leading to:

$$
\left(\lambda^{2}[M]+\lambda[C]+[K]\right)\{\delta\}=\{0\}
$$

In the case of a rotating dynamic system the argument $\lambda=i \omega$ and $\{\delta\}$ is a complex vector, consisting of real and imaginary part, Eq. (23). The resulting matrix equation specified in the real domain is of double size, (24), with a symmetric total matrix as shown in the previous section.

If a damped dynamic system is considered, one has to set $\lambda=v+i \omega$, where $v$ is the damping coefficient and $\omega$ is the natural frequency. The displacement vector $\{\delta\}$ is also complex, Eq. (23). In this case the matrix equation of motion transformed into the real domain takes the form:

$$
\left[\begin{array}{cc}
{[K(\omega, v)]} & \omega\left(2 v[M]+[C]_{d}\right) \\
-\omega\left(2 v[M]+[C]_{d}\right) & {[K(\omega, v)]}
\end{array}\right]\left\{\begin{array}{l}
\{\delta\}_{R e} \\
\{\delta\}_{\text {Im }}
\end{array}\right\}=\{0\}
$$

where:

$$
[K(\omega, v)]=\left(\omega^{2}-v^{2}\right)[M]+v[C]_{d}+[K]
$$

is the dynamic stiffness matrix. Since all submatrices in Eq. (37) are symmetric, the total matrix is asymmetric.

In most dynamic systems $v<<\omega$ and first $\omega_{0}$ for an undamped system, $v=0$, can be determined by setting $\operatorname{Det}\left(\left[K\left(\omega_{0}, v\right)\right]\right)=0$. After that, $v$ can be obtained for the known $\omega_{0}$ from a condition $\operatorname{Det}\left(\left[K\left(\omega_{0}, v\right)\right]\right)=0$. The value $\omega^{*}=\sqrt{\omega_{0}^{2}-v^{2}}$ is well-known as the natural frequency of damped dynamic system. If $v<\omega$, the eigenvalue problem (30) with two unknown parameters can be solved by employing an iterative numerical procedure.

Finally, one can conclude that in the original formulation in the complex domain, the Coriolis mass matrix is antisymmetric, whereas the damping matrix is symmetric. In the real domain it is the other way around.

\section{NON-LINEAR STRAINS}

\subsection{FORMULATION OF NON-LINEAR STRAINS}

For the second order non-linear strains, Green-Lagrange formulation is ordinarily used, [12]. However, another formulation of the non-linear strain-displacement relationships, called the engineering strains has been proposed in [13]. It is related to large local displacements and rotations but small strains. The local rigid body displacements from the total displacements 
are removed and small local displacement field is used to derive objective local strains. Differences between the two formulations can be illustrated in a simple case of a flat membrane.

a)

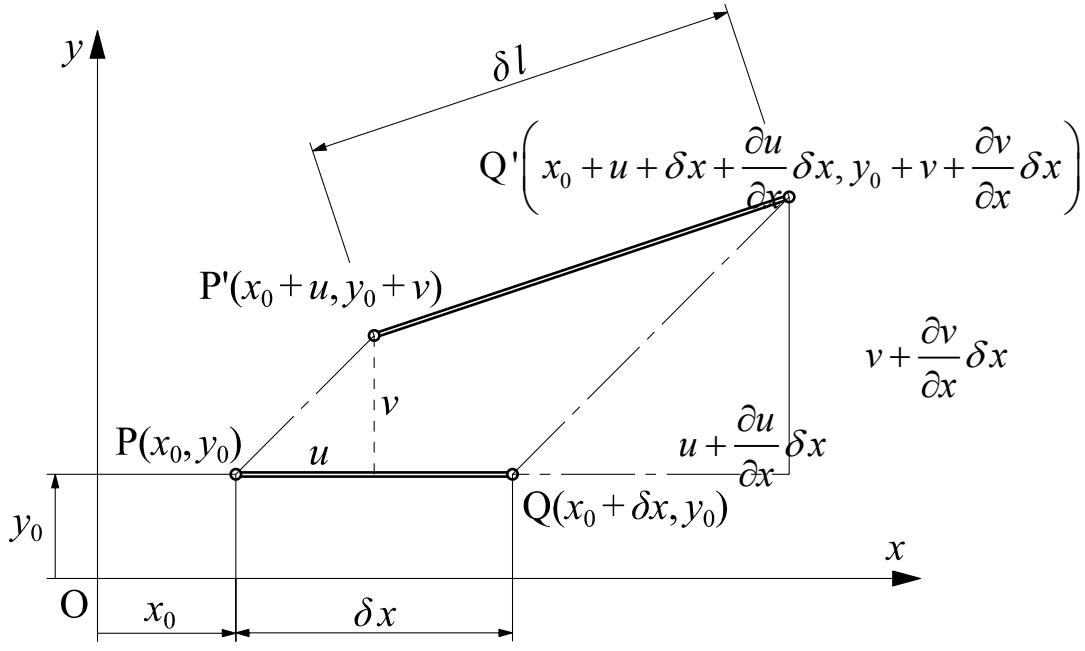

b)

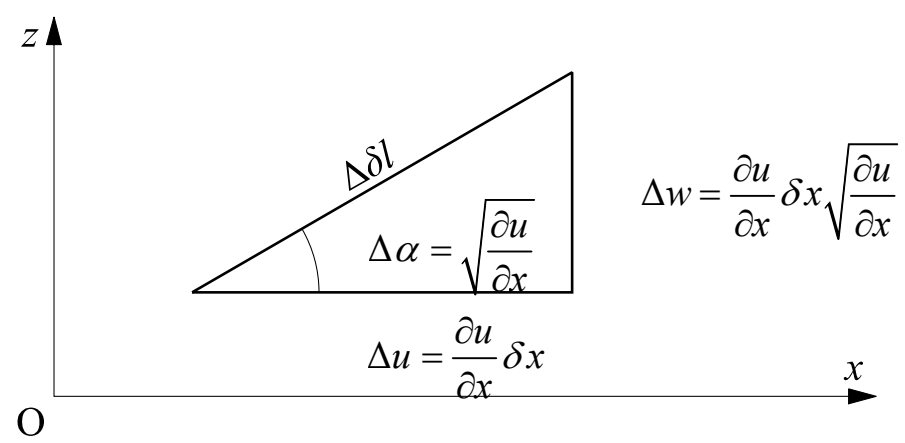

Fig. 6 Consideration of non-linear strain: a) stretching of a membrane filament, b) virtual local rotation

In Figure 6 a membrane filament of length $\delta_{x}$, determined with the end points $P$ and $Q$, is shown. Due to the in-plane deformation prescribed with displacements $u$ and $v$, these points are moved to a new position $P^{\prime}$ and $Q^{\prime}$ with the infinitesimal increase of coordinates and filament length. The strain is defined as:

$$
\varepsilon=\frac{\delta l-\delta x}{\delta x}
$$

where:

$$
\delta l=\delta x \sqrt{\left(1+\frac{\partial u}{\partial x}\right)^{2}+\left(\frac{\partial v}{\partial x}\right)^{2}}
$$

Hence, Eq (40) takes the form:

$$
\varepsilon=\sqrt{\left(1+\frac{\partial u}{\partial x}\right)^{2}+\left(\frac{\partial v}{\partial x}\right)^{2}}-1
$$

Eq. (41) can be written as:

$$
\varepsilon=\sqrt{1+\eta}-1
$$

where: 


$$
\eta=2 \frac{\partial u}{\partial x}+\left(\frac{\partial u}{\partial x}\right)^{2}+\left(\frac{\partial v}{\partial x}\right)^{2}
$$

Expanding the square root in (42) into power series, yields:

$$
\varepsilon=\frac{1}{2} \eta-\frac{1}{8} \eta^{2}+O\left(\eta^{3}, \eta^{4}, \ldots\right)
$$

Truncating the higher-order terms in (44), the application of the first term and Eq. (43) leads to:

$$
\varepsilon=\frac{\partial u}{\partial x}+\frac{1}{2}\left[\left(\frac{\partial u}{\partial x}\right)^{2}+\left(\frac{\partial v}{\partial x}\right)^{2}\right]
$$

The first term in (45) is the linear strain, while the second term is the non-linear GreenLagrange strain, [12].

If the first two terms of Eq. (44) are taken into account and the first and second derivatives are retained, two terms $\left(\frac{\partial u}{\partial x}\right)^{2}$ of opposite sign occur, which are cancelled and one arrives at:

$$
\varepsilon_{E}=\frac{\partial u}{\partial x}+\frac{1}{2}\left(\frac{\partial v}{\partial x}\right)^{2}
$$

The second term in (46) represents the engineering non-linear strain, [13].

The term $\frac{1}{2}\left(\frac{\partial u}{\partial x}\right)^{2}$ in Eq. (45) can be imagined as a virtual local infinitesimal rotation induced by variation of axial displacement, as shown in the zoomed view in Figure 6b. This assumption is acceptable since:

$$
\Delta \delta I=\sqrt{\Delta u^{2}+\Delta v^{2}}=\left[\frac{\partial u}{\partial x}+\frac{1}{2}\left(\frac{\partial u}{\partial x}\right)^{2}\right] \delta x
$$

The virtual rotation is not present in the engineering strain, Eq. (46).

The second equation of the Green-Lagrange strain for a membrane in $y$-direction can be written analogue to the first equation (45). Then both equations can be extended to the case of a plate with deflection $w,[12]$ :

$$
\begin{aligned}
& \varepsilon_{x G}=\frac{\partial u}{\partial x}+\frac{1}{2}\left[\left(\frac{\partial u}{\partial x}\right)^{2}+\left(\frac{\partial v}{\partial x}\right)^{2}+\left(\frac{\partial w}{\partial x}\right)^{2}\right] \\
& \varepsilon_{y G}=\frac{\partial v}{\partial y}+\frac{1}{2}\left[\left(\frac{\partial v}{\partial y}\right)^{2}+\left(\frac{\partial u}{\partial y}\right)^{2}+\left(\frac{\partial w}{\partial y}\right)^{2}\right]
\end{aligned}
$$

The engineering strains are obtained by neglecting the first terms in the brackets in (48).

Based on the above considerations it is obvious that the Green-Lagrange strains are objective measures, while the engineering strains are geometric measures. The former formulation is neither directly measurable, nor geometrically interpretable. Moreover, it does not have a defined direction. It is simply an energy-related function, [13]. 


\subsection{GREEN - LAGRANGE STRAINS}

In order to avoid complicated transformation of the orthogonal coordinates to curvilinear ones, the analogy of physical meaning of different terms in (48) is used for formulation of the second-order Green-Lagrange strains (GLS). Accordingly, one can write for a toroidal shell:

$$
\begin{aligned}
& \varepsilon_{\vartheta}^{*}=\frac{1}{2}\left[\varepsilon_{\vartheta}^{2}+\left(\varepsilon_{\vartheta \varphi}^{(1)}\right)^{2}+\beta_{\vartheta}^{2}\right] \\
& \varepsilon_{\varphi}^{*}=\frac{1}{2}\left[\varepsilon_{\varphi}^{2}+\left(\varepsilon_{\vartheta \varphi}^{(2)}\right)^{2}+\beta_{\varphi}^{2}\right]
\end{aligned}
$$

where $\varepsilon_{\vartheta}, \varepsilon_{\varphi}$ and $\varepsilon_{\vartheta \varphi}$ are linear tensional and in-plane shear strains, and $\beta_{\vartheta}$ and $\beta_{\varphi}$ are angles of shell rotation, [9]. Shear stain $\varepsilon_{\vartheta \varphi}$ is split into two parts, one for the meridional and another one for the circumferential direction.

The strain energy due to pre-stressing can be presented in the form:

$$
E_{G}=\int_{0}^{2 \pi} \int_{0}^{2 \pi}\left(\varepsilon_{\vartheta}^{*} N_{\vartheta}+\varepsilon_{\varphi}^{*} N_{\varphi}\right) \operatorname{ard} \vartheta d \varphi
$$

Taking into account Eq. (49) and (1) one obtains after integration per circumferential coordinate $\varphi$, condensed energy on the shell cross-section:

$$
\begin{aligned}
E_{G}= & \int_{\vartheta}\left[\frac{1}{2} c_{1}\left(U^{\prime}\right)^{2}+\frac{1}{2} c_{2} U^{2}+\frac{1}{2} c_{3}\left(V^{\prime}\right)^{2}+\frac{1}{2} c_{4} V^{2}+c_{5} V^{\prime} V\right. \\
& \left.+\frac{1}{2} c_{6}\left(W^{\prime}\right)^{2}+\frac{1}{2} c_{7} W^{2}+c_{8} U V+c_{9}\left(U^{\prime} W-U W^{\prime}\right)+c_{10} U W+c_{11} V W\right] d \vartheta,
\end{aligned}
$$

where $c_{i}(\vartheta), i=1,2 \ldots 11$ are variable coefficients given in Appendix.

\subsection{ENGINEERING STRAINS}

According to Eqs. (45) and (46) the engineering strains are defined by omitting the first terms in Green - Lagrange formulation, Eqs. (49). Hence, one can write:

$$
\begin{aligned}
& \tilde{\varepsilon}_{\vartheta}=\frac{1}{2}\left[\left(\varepsilon_{\vartheta \varphi}^{(1)}\right)^{2}+\beta_{\vartheta}^{2}\right] \\
& \tilde{\varepsilon}_{\varphi}=\frac{1}{2}\left[\left(\varepsilon_{\vartheta \varphi}^{(2)}\right)^{2}+\beta_{\varphi}^{2}\right]
\end{aligned}
$$

Consequently some coefficients $c_{i}(\vartheta), i=1,2 \ldots 11$ in the strain energy formulation, Eq. (51), are equal to zero and some of them are reduced, as shown in Appendix. 
I Senjanović, N. Alujević, I. Ćatipović, D. Čakmak, N. Vladimir, Ž. Lozina: Some dilemmas in energy approach to vibration and stability analysis of pressurized and rotating toroidal shells

\subsection{ILLUSTRATIVE EXAMPLES}

\subsubsection{VIBRATION OF TOROIDAL SHELL}

Closed toroidal shell with the following geometric and physical parameters is considered, Figure 1: $R=1 \mathrm{~m}, a=0.4 \mathrm{~m}, h=0.01 \mathrm{~m}, E=2.1 \times 10^{11} \mathrm{~N} / \mathrm{m}^{2}, v=0.3, \rho=7850 \mathrm{~kg} / \mathrm{m}^{3}$. Calculation of natural vibration is performed by RRM, using $2 \times 15$ Fourier terms per one displacement, for the Green-Lagrange strain. Natural frequencies are shown in Figs. 2 and 3, as a function of dimensionless rotation speed, $\Omega / \omega_{0}$, [9]. Natural frequencies determined by FEM software NASTRAN are also included in Figs. 2 and 3, [14]. Small differences between the RRM and FEM results can be noticed.

The same problem is analysed by taking the engineering strain into account, Eqs. (52), utilizing the corresponding variable coefficients $c_{i}(\vartheta)$ in the geometric stiffness matrix from Appendix. Only values for $\Omega / \omega_{0}=1$ are indicated in Figs. 2 and 3 with arrows, in order to avoid clustering of many lines. It is evident that natural frequencies determined with the engineering strain are somewhat higher than those obtained with the Green-Lagrange strain. Generally speaking, the influence of the engineering strain on vibrations is quite small since contribution of geometric stiffness to general shell stiffness in the considered case is small, Eq. (13).

\subsubsection{BUCKLING OF TOROIDAL SHELL}

The governing eigenvalue problem is deduced from Eq. (13) and reads:

$$
\left([K]-p[G]_{p}\right)\{\delta\}=\{0\}
$$

Geometric stiffness matrix plays the main role in the case of shell buckling like the mass matrix does in vibration.

Stability of the same toroidal shell as in the previous example is analysed next. The values of the buckling parameter $\lambda=p_{c r} / p$, determined by the RRM taking the Green-Lagrange strains into account, Eqs. (49), are listed in Table 1.

Buckling analysis is also performed by using the engineering strains, Eqs. (52). The eigenvalue spectrum starts with extremely large negative values, growing to the positive scale. Physical meaning of the negative buckling parameter means the change of external to internal pressure, i.e. from unstable to stable domain. Hence, the minimum positive values of buckling parameter, which are shown in Table 1, are relevant for the loss of shell stability. By comparing the obtained results in Table 1, it is obvious that the values of buckling parameter determined by using the engineering strains are highly overestimated.

Table 1 Buckling parameter $\lambda=p_{c r} / p$, closed toroidal shell, $R=1 \mathrm{~m}, a=0.4 \mathrm{~m}, h=0.01 \mathrm{~m}, p=1 \mathrm{MPa}$

\begin{tabular}{|c|c|c|c|c|c|c|}
\hline \multirow[b]{2}{*}{ Mode No. } & \multirow[b]{2}{*}{ Mode type } & \multirow[b]{2}{*}{$n$} & \multicolumn{2}{|c|}{$R R M, 2 \times 15$ terms } & \multicolumn{2}{|c|}{$F E M$} \\
\hline & & & $G L S$ & $E S, \varepsilon(\%)$ & $\begin{array}{c}\text { Abaqus } \\
\text { S8R5 } \\
50 \times 124, \varepsilon(\%)\end{array}$ & $\begin{array}{c}\text { Catia } \\
\text { QD } 8 \\
50 \times 124, \varepsilon(\%)\end{array}$ \\
\hline 1 & Asym. & 0 & 3.850 & $6.597(71.35)$ & $4.083(6.05)$ & $3.869(0.49)$ \\
\hline 2 & Asym. & 2 & 4.545 & $7.176(57.89)$ & $4.384(-3.54)$ & $4.570(0.55)$ \\
\hline 3 & Sym. & 2 & 4.687 & $7.382(57.50)$ & $4.386(-6.42)$ & $4.756(1.47)$ \\
\hline 4 & Asym. & 3 & 5.915 & $7.398(25.07)$ & $5.565(-5.92)$ & $5.842(-1.23)$ \\
\hline 5 & Sym. & 3 & 5.921 & $7.409(25.13)$ & $5.582(-5.72)$ & $5.863(-0.98)$ \\
\hline
\end{tabular}




\begin{tabular}{|l|l|l|l|l|l|l|}
\hline 6 & Asym. & 4 & 7.712 & $8.838(14.60)$ & $7.371(-4.42)$ & $7.597(-1.49)$ \\
\hline 7 & Asym. & 4 & 7.713 & $8.839(14.60)$ & $7.373(-4.41)$ & $7.598(-1.49)$ \\
\hline
\end{tabular}

For illustration values of buckling parameter $\lambda$ determined for $n=2$ for the Green-Lagrange strains, semi-engineering strains ignoring only dominant non-linear terms $\left(\frac{\partial u}{\partial \vartheta}\right)^{2}$ and $\left(\frac{\partial v}{\partial \varphi}\right)^{2}$ in (51), and full engineering strains, are shown in Table 2. A large number of negative values is observed due to the reduction of coefficients $c_{i}(\vartheta)$. Since the structure of both geometric stiffness matrices is very similar, one could not expect such a constellation of the results. This fact is a result of coupling between shell tensional displacements and deflection, manifested via their products in the energy equation (51).

Table 2 Buckling parameter, $\lambda=p_{c r} / p, R R M, 2 \times 15$ terms, $n=2$

\begin{tabular}{|c|c|c|c|c|c|c|c|c|}
\hline $\begin{array}{c}\text { Ordinary } \\
\text { number }\end{array}$ & -28 & -27 & -2 & -1 & 1 & 2 & 3 & 4 \\
\hline GLS & & & & & 4.545 & 4.687 & 16.043 & 16.049 \\
\hline Semi-ES & $-4.338 \cdot 10^{5}$ & $-4.338 \cdot 10^{5}$ & -2475.3 & -905.6 & 5.703 & 5.894 & 16.709 & 16.712 \\
\hline$E S$ & $-4.244 \cdot 10^{5}$ & $-4.244 \cdot 10^{5}$ & -553.6 & -364.5 & 7.176 & 7.382 & 17.414 & 17.418 \\
\hline
\end{tabular}

In addition the same buckling problem is solved by FEM. Two commercial software packages are used, i.e. Abaqus [15] and Catia [16]. The applied finite element types and finite element mesh density are indicated in Table 1 . Comparison of discrepancies between FEM results and RRM results, $\varepsilon(\%)$, listed in Table 1 shows that the Catia values agree somewhat better with the RRM results than the Abaqus values. In the considered example savings in the CPU time by employing RRM comparing to FEM is ca 1:10. It is interesting to point out that the buckling modes of the closed toroidal shell are identical to its natural vibration modes shown in [9].

\section{CONCLUSION}

Doubts in vibration analysis of rotating and pressurized toroidal shells concerning physical meaning of the strain and kinetic energy terms of different displacement order, symmetry of Coriolis mass matrix, and reliability of different non-linear strain formulations, are herein made clear.

The energy terms depend on different order of displacement field, $E\left(\delta^{i}\right), i=1,2,3,4$. Linearized non-linear strain energy and the accompanying kinetic energy of zero order, $E_{a k}^{0}=E_{G}\left(\delta^{0}\right)+E_{k}\left(\delta^{0}\right)$, are actually the energy accumulated due to the shell rotation. Their difference in the energy balance equation, $\Pi\left(\delta^{0}\right)=E_{G}\left(\delta^{0}\right)-E_{k}\left(\delta^{0}\right)$, is constant, and vanishes in the energy variation. The first order terms, $\Pi\left(\delta^{1}\right)=E_{G}\left(\delta^{1}\right)-E_{k}\left(\delta^{1}\right)=0$, disappear a priori in the energy balance equation. The energy terms of higher order, $E_{G}\left(\delta^{3}\right)$ and $E_{G}\left(\delta^{4}\right)$, are small quantities omitted in the linearization of the vibration problem at hand. In such a way the energy balance equation is reduced to the ordinary second order terms i.e. 
$\Pi\left(\delta^{2}\right)=E_{S}\left(\delta^{2}\right)+E_{G}\left(\delta^{2}\right)-E_{k}\left(\delta^{2}\right)$. The solution to the problem is obtained by minimizing the value of $\Pi\left(\delta^{2}\right)$ for assumed natural vibration modes.

By employing the Rayleigh-Ritz method for the above purpose, symmetric Coriolis mass matrix is obtained as a result of the energy approach, and a common space and time argument, $n \varphi+\omega t$, of Fourier series. Since this is not possible in development of general double curved shell finite element, the governing matrix differential equation of motion is obtained in the complex form with antisymmetric Coriolis mass matrix. However, if the complex matrix equation is transferred into real domain, its size is doubled and the total Coriolis mass matrix takes a symmetric form. Direct comparison of the well-known antisymmetric Coriolis matrix in the complex equation of motion with symmetric Coriolis matrix in the real equation of motion, derived by employing the energy approach, raises some doubt and confusion, which are eliminated in this way.

Concerning the application of the Green-Lagrange non-linear strain formulation and the engineering strain formulation, it is observed that the difference in their influence on vibration is minor. This is because the contribution of geometric stiffness to the total stiffness in the elastic domain is small. However, in buckling analysis, the geometric stiffness matrix plays a main role, just like the mass matrix does in vibration analysis. In spite of the fact that the general formulation of the engineering strains seems to be physically based, this is not applicable for shell buckling analysis due to the highly overestimated critical pressure. This results from strong coupling between the shell in-surface displacements and deflection. Such coupling is not present in the plate theory, and therefore the Green-Lagrange strains and the engineering strains present almost the same results.

The Green-Lagrange strain formulation is energy-related and pre-eminent for applications in energy approach to shell dynamic and stability analyses.

\section{ACKNOWLEDGEMENT}

This investigation has received funding within the international collaborative project Global Core Research Center for Ships and Offshore Plants (GCRC-SOP, No. 2011-0030669), established by the Republic of Korea Government (MSIP) through the National Research Foundation of South Korea (NRF).

\section{REFERENCES}

[1] V.V. Novozhilov, Thin Shell Theory, P. Noordhoff, Groningen, The Netherlands, 1964. https://doi.org/10.1007/978-94-017-5352-4

[2] W. Soedel, Vibrations of Shells and Plates (3 ${ }^{\text {rd }}$ edition), New York, Marcel Dekker, Inc. 2004.

[3] I. Senjanović, Theory of Shells of Revolution, Ship Research Institute, Zagreb, Croatia, 1972.

[4] O.C. Zienkiewicz, The Finite Element Method in Engineering Science, McGraw - Hill, London, 1971. 
[5] K.J. Bathe, Finite Element Procedures, Prentice Hall, Upper Saddle River, New Jersey, 1996.

[6] N. Alujević, N. Campillo-Davo, P. Kindt, W. Desmet, B. Pluymers, S. Vercammen, Analytical solution for free vibrations of rotating cylindrical shells having free boundary conditions, Engineering structures, Vol. 132, pp. 152-171, 2017.

https://doi.org/10.1016/j.engstruct.2016.11.008

[7] S. Sun, D. Cao, Q. Han, Vibration studies of rotating cylindrical shells with arbitrary edges using characteristic orthogonal polynomials in the Rayleigh-Ritz method, International Journal of Mechanical Sciences, Vol. 68, pp. 180-189, 2013.

https://doi.org/10.1016/j.ijmecsci.2013.01.013

[8] I. Senjanović, I. Ćatipović, N. Alujević, N. Vladimir, D. Čakmak, A finite strip for the vibration analysis of rotating cylindrical shells, Thin-Walled Structures, Vol. 122, pp. 158172, 2018. https://doi.org/10.1016/j.tws.2017.10.017

[9] I. Senjanović, N. Alujević, I. Ćatipović, D. Čakmak, N. Vladimir, Vibration analysis of rotating toroidal shell by the Rayleigh-Ritz method and Fourier series, Engineering Structures, Vol. 173, pp. 870-891, 2018.

https://doi.org/10.1016/i.engstruct.2018.07.029

[10] I. Senjanović, I. Ćatipović, N. Alujević, D. Čakmak, N. Vladimir, A finite strip for the vibration analysis of rotating toroidal shell under internal pressure, ASME Journal of Vibration and Acoustics, Vol. 141, No. 2, pp. 021013-021013-17, 2019.

https://doi.org/10.1115/1.4041734

[11] I. Senjanović, I. Ćatipović, N. Alujević, D. Čakmak, N. Vladimir, Free in-plane and out-ofplane vibration of rotating thin ring based on the toroidal shell theory, Arch. Mech., Vol. 70, No. 5, pp. 429-455, 2018.

[12] M.A. Crisfield, Non-Linear Finite Element Analysis of Solids and Structures, Volume 1: Essentials, John Wiley \& Sons, Chichester, West Sussex, England, 1991.

[13] P.F. Pai, A.H. Nayfeh, A new method for the modeling of geometric nonlinearities in structures, Computers \& Structures, Vol. 53, No. 4, pp. 877-895, 1994.

https://doi.org/10.1016/0045-7949(94)90376-X

[14] MSC.MD NASTRAN 2010, Dynamic Analysis User's Guide, MSC Software, 2010.

[15] Dassault Systèmes, Abaqus 6.9 User's guide and theoretical manual, Hibbitt, Karlsson \& Sorensen, Inc. 2009.

[16] Dassault Systèmes, Catia V5R19 Documentation: Finite Element Reference Guide, 2007. 


\section{APPENDIX A}

\section{VARIABLE COEFFICIENTS OF THE STRAIN ENERGY DUE TO PRE-STRESSING}

1. GREEN-LAGRANGE STRAIN

$$
\begin{aligned}
& c_{1}=c_{3}=c_{6}=c_{9}=\pi \frac{r}{a} N_{\vartheta} \\
& c_{2}=\pi\left[\frac{r}{a} N_{\vartheta}+\frac{a}{r}\left(n^{2}+\cos ^{2} \vartheta\right) N_{\varphi}\right] \\
& c_{4}=\pi \frac{a}{r}\left[\cos ^{2} \vartheta N_{\vartheta}+\left(n^{2}+\sin ^{2} \vartheta\right) N_{\varphi}\right] \\
& c_{5}=-\pi \cos \vartheta N_{\vartheta} \\
& c_{7}=\pi\left[\frac{r}{a} N_{\vartheta}+\frac{a}{r}\left(n^{2}+\sin ^{2} \vartheta\right) N_{\varphi}\right] \\
& c_{8}=\pi \frac{a}{r} n \cos \vartheta N_{\varphi} \\
& c_{10}=\pi \frac{a}{r} \sin \vartheta \cos \vartheta N_{\varphi} \\
& c_{11}=2 \pi \frac{a}{r} n \sin \vartheta N_{\varphi} .
\end{aligned}
$$

\section{ENGINEERING STRAIN}

$$
\begin{aligned}
& c_{1}=0, \quad c_{3}=c_{6}=c_{9}=\pi \frac{r}{a} N_{\vartheta} \\
& c_{2}=\pi\left(\frac{r}{a} N_{\vartheta}+\frac{a}{r} n^{2} N_{\varphi}\right) \\
& c_{4}=\pi \frac{a}{r}\left(\cos ^{2} \vartheta N_{\vartheta}+\sin ^{2} \vartheta N_{\varphi}\right) \\
& c_{5}=-\pi \cos \vartheta N_{\vartheta} \\
& c_{7}=\pi \frac{a}{r} n^{2} N_{\varphi} \\
& c_{8}=c_{10}=0 \\
& c_{11}=\pi \frac{a}{r} n \sin \vartheta N_{\varphi} .
\end{aligned}
$$

